\title{
Etnografía institucional como aproximación al habitar cotidiano ${ }^{1}$.
}

\section{Paola Jirón Martínez²; Nicolás Orellana Águila³; Walter Imilán ${ }^{4}$}

\author{
Fecha de recepción: 30 de julio de 2018 \\ Fecha de aprobación: 03 de octubre de 2018
}

\begin{abstract}
Resumen
El presente artículo indaga en el enfoque de investigación y metodología de etnografía institucional (EI), la que se centra en develar la forma en que las personas vivencian procesos institucionales de control en la vida cotidiana, cómo se relacionan con complejos institucionales y cómo moldean el continuo de sus vidas cotidianas. Con este fin, la EI es un modo de acercamiento a los fenómenos sociales que toma como punto de partida las experiencias cotidianas, situadas y incorporadas. Surgida desde la sociología feminista, hasta ahora la EI no se ha aplicado a las relaciones entre habitantes e intervenciones urbanas, ni tampoco de manera explícita en América Latina. El objetivo del presente artículo es explorar las posibilidades metodológicas de la EI en contextos urbanos, expandiendo de esta manera la creciente exploración metodológica en la investigación urbana. Con base en el trabajo de campo que se realiza actualmente en la ciudad de Santiago de Chile, se explica cómo la EI puede develar maneras en que las relaciones de control en las intervenciones urbanas (programas y proyectos urbanos específicos) muchas veces dominan e ignoran conocimientos locales y situados.
\end{abstract}

Palabras clave: etnografía institucional, experiencias cotidianas, habitar urbano, problemática, relaciones de control.

Este artículo se realizó en el marco del Proyecto FONDECYT N ${ }^{\circ} 1171554$ "Prácticas de intervenir y habitar el territorio: develando el conocimiento urbano situado" y del Núcleo Milenio Movilidades y Territorios MOVYT.

2 Doctora en Planificación Urbana y Regional LSE. Profesora Asociada del Instituto de la Vivienda, Universidad de Chile. Santiago, Chile. Contacto: paolajiron@ uchilefau.cl

3 Doctor en Ciencias Políticas y Sociales, Université Catholique de Louvain. Profesor Universidad Bernardo O’Higgins. Santiago, Chile. Contacto: orellanani@ docente.ubo.cl

4 Doctor en Planificación TU Berlin. Investigador del Centro de Estudios Arquitectónicos, Urbanísticos y del Paisaje, Universidad Central de Chile. Santiago, Chile. Contacto: walter.imilan@ucentral.cl 


\title{
Institutional ethnography as an approach to daily life
}

\begin{abstract}
This article explores the research and methodology approach of Institutional Ethnography (IE). The IE focuses on unveiling the way in which people experience institutional control processes in everyday life, how subjects relate to institutional complexes, and how they shape the continuum of their daily lives. To this end, the IE is a way of approaching social phenomena that takes everyday experiences as a starting point, situated and embodied. Emerged from the feminist sociology, until now the IE has not been applied to relations among inhabitants and urban interventions, nor explicitly in Latin America.

The objective of this article is to explore methodological possibilities for IE in urban contexts, thus expanding the growing methodological exploration in urban research. Based on fieldwork that is currently carried out in the city of Santiago de Chile, it explains that the IE can reveal how the control relationships in urban interventions (specific urban programs and projects) often dominate and ignore local and located knowledge.
\end{abstract}

Keywords: Institutional ethnography - daily experiences - urban living problematic - control relations.

\section{Etnografia institucional como aproximaçâo ao habitar cotidiano}

\begin{abstract}
Resumo
O presente artigo indaga na perspectiva de pesquisa e metodologia de Etnografia Institucional (EI). A EI centra-se em desvendar a forma em que as pessoas vivenciam processos institucionais de controle na vida cotidiana, de como se relacionam os sujeitos com complexos institucionais, e de como eles moldam o contínuo de suas vidas cotidianas. Com este fim, a EI é um modo de aproximação aos fenômenos sociais que toma como ponto de partida as experiências cotidianas, situadas e encorporadas. Surgida desde a sociologia feminista, a EI até agora não tem sido aplicada às relações entre habitantes e intervenções urbanas, nem de maneira explícita na América Latina.

O objetivo do presente artigo é explorar as possibilidades metodológicas da EI e contextos urbanos expandindo desta maneira a crescente exploração metodológica na pesquisa urbana. Com base no trabalho de campo que é realizado atualmente na cidade de Santiago do Chile, explica-se como a EI pode revelar maneiras em que as relações de controle nas intervenções urbanas (programas e projetos urbanos específicos), muitas vezes dominam e ignoram conhecimentos locais e situados.
\end{abstract}

Palavras-chave: etnografia institucional - experiências cotidianas - habitar urbano - problemática - relações de controle. 


\section{Introducción}

El presente artículo indaga en el enfoque de investigación y metodología de Etnografía Institucional (EI) como un modo de acercamiento teórico y metodológico a los fenómenos sociales, tomando como punto de partida las experiencias cotidianas, situadas y encorporadas. En particular, la EI se ha desarrollado para develar la manera en que las personas vivencian procesos institucionales de control en la vida cotidiana, cómo los sujetos se relacionan con complejos institucionales y cómo éstos moldean el continuo de sus vidas cotidianas. Siguiendo a Smith (2006, p. 3), la EI "no pretende ser una manera de descubrir el mundo cotidiano como tal, sino de mirar más allá de lo cotidiano para descubrir cómo llegó a suceder como lo hace". La EI plantea una manera de analizar los fenómenos sociales explicitando a los investigadores en el lugar que están situados, y haciendo de la "experiencia directa del mundo cotidiano el terreno primario de su conocimiento" (Smith, 1974, p. 11).

La EI pone énfasis en una forma de generar conocimiento con foco en la experiencia de los sujetos, que es siempre localizada y particular (Smith, 1987). De acuerdo con Dorothy Smith (1992), la EI es un modo de acercamiento que toma un "diferente punto de partida" (standpoint), que comienza en la experiencia cotidiana para, a partir de ella, identificar las problemáticas de investigación, entendidas como un desajuste entre lo que es experimentado por las personas en su cotidianidad y la abstracción que se realiza sobre ellas por medio de un régimen institucional que produce relaciones de control. En definitiva, el objetivo es rastrear cómo se moldean las vidas de las personas (Deveau, 2008).

De esta forma, la EI se diferencia de la Etnografía de las instituciones, desarrollada desde la Antropología en trabajos como los de Shore (2000), Fassin (2013 [2011]) o Lins-Ribeiro (2001), ya que esta última considera a las instituciones en sí mismas como locus del trabajo de campo etnográfico, indagando los efectos que una 
organización singular tiene sobre las experiencias de individuos concretos. Mientras que la EI rastrea, a partir de la experiencia situada, cómo opera un régimen institucional amplio, modelando translocalmente esas experiencias, a través de la generación de relaciones de control que operan simultáneamente en diferentes localizaciones.

La EI toma su impulso desde una Sociología feminista (Smith, 1992, 1990 y 1987) y posteriormente se propaga a una variedad de campos y disciplinas de investigación, principalmente en torno a la crianza (Griffith y Smith, 2004; Griffith, 1995), la salud (Campbell, 2014; Paulsen, 2015; Ng et al., 2013; Rankin y Campbell, 2006 y 2009) y la educación (Griffith y Smith 2004; Grahame, 1998; O'Neill, 1998; Griffith, 1995), así como se ha desarrollado con bastante éxito en diversos contextos debido a su capacidad explicativa y comprensiva. Sin embargo, la amplia literatura en EI, principalmente en inglés, aún es poco trabajada en América Latina y adaptada a procesos locales, menos aún se ha aplicado para comprender el accionar de intervenciones urbanas.

Este artículo tiene dos propósitos: por un lado, presentar los puntos fundamentales de análisis de esta estrategia como herramienta de investigación y, en segundo término, explorar una puesta en práctica en el campo de los estudios urbanos ${ }^{5}$.

La EI se ha implementado en el marco del proyecto de investigación "Prácticas
de intervenir y habitar el territorio: develando el conocimiento urbano situado",
el cual intenta comprender la producción de conocimientos que surgen a partir
de la interacción entre prácticas de intervención urbana y prácticas cotidianas de
habitar que dan forma al territorio en Santiago de Chile. El proyecto plantea que las
transformaciones políticas y económicas acaecidas en el contexto de la globalización
y la profundización del modelo neoliberal en Chile, se han caracterizado por
imponer una planificación urbana tecnocrática, centralista y vertical en la toma de
decisiones, que, si bien ha generado significativos adelantos materiales en la ciudad,
por medio de la provisión de vivienda, infraestructura y equipamiento urbano, estas
intervenciones reconocen, en menor medida, las dinámicas cotidianas de habitar la
ciudad. Por otro lado, muchas de las intervenciones urbanas son adopciones de
modelos internacionales considerados exitosas. Así, la circulación de modelos de
políticas (globales) juega un rol central en cómo se piensan las intervenciones locales
(McCann y Ward 2011, 2012a y 2012b; Delgadillo, 2014; Montero 2016 y 2017). La
preocupación teórica por este tipo de procesos ya ha estado presente en el debate de 
La ciudad es habitada por medio de una diversidad de prácticas cotidianas que se contraponen, resisten y subvierten el conocimiento experto sobre esta, a partir de diversos saberes y conocimientos de carácter cotidiano (Jirón y Mansilla, 2014). No obstante, las intervenciones urbanas se sustentan hegemónicamente en conocimientos expertos de carácter tecnocientífico de orden positivista, que tiende a generar un impacto directo sobre la territorialidad de los habitantes, moldeando la experiencia de habitar la ciudad a través de su fragmentación espacial y temporal.

El presente artículo se basa, primeramente, en una investigación bibliográfica que da cuenta de los propósitos, objetivos y alcances, tanto teóricos como aplicados, de este método, dando cuenta de sus principales giros epistemológicos, ontológicos y conceptuales; después, se presentan algunos principios procedimentales de la EI, además de los campos de estudio en los que se ha aplicado. En segundo lugar, basado en información empírica de trabajo de campo etnográfico en desarrollo en un barrio de Santiago, se propone un ejercicio de análisis que explora en las tensiones entre la vida cotidiana de los habitantes y las intervenciones de un programa de mejoramiento barrial ${ }^{6}$. En el caso de la investigación en la que se basa este artículo, el trabajo de campo aún se encuentra en desarrollo, por lo que se presenta la implementación de la primera fase de la metodología propuesta.

Es importante destacar que la EI es un método que puede ser complementado con otras estrategias de investigación etnográfica, que han mostrado interesantes posibilidades en búsqueda de una perspectiva situada en el campo de los estudios urbanos, tales como las etnografías móviles y multisituadas (Jirón, 2011;

los estudios urbanos en obras de Jacobs (1967), Lefebvre (1972 y 1973), y De Certeau (2000), así como en los trabajos de Robinson (2006) y Roy (2011), preocupadas respecto de la manera en que se adapta y adoptan las teorías mundiales.

6 El proyecto "Prácticas de intervenir y habitar el territorio: develando el conocimiento urbano situado", analiza intervenciones públicas en el territorio de diferente tipo: barriales, de transporte, en el ámbito de cultura y de gestión de información. Para mayor información ver: www.santiagosemueve.com 
Imilán et al., 2015; Spinney, 2011), las etnografías políticas (Auyero, 2012), e incluso las autoetnografías (Iturra, 2014).

\section{De la experiencia a la Etnografía Institucional. Hacia un nuevo terreno del conocer}

A mediados de la década del 2000, Dorothy Smith, pionera y fundadora de la perspectiva de la Etnografía Institucional (Billo y Mountz, 2015; Luken y Vaughan, 2015; Campbell, 2014; Grahame, 1998), publicó una obra titulada Institutional Ethnography: A Sociology for the People (Smith, 2005), y editó el libro Institutional Ethnography as practice (Smith, 2006). Ambos textos se establecen como corolarios de un desarrollo que tiene sus inicios en la década de 1980, y cuyo objetivo fundamental es explorar y explicar la organización social del conocimiento para dilucidar cómo las actividades y experiencias cotidianas de las personas están coordinadas, postulando "una sociología que no comienza en la teoría, sino en la experiencia de las personas" (Smith, 2006, p. 2).

Siguiendo a Smith, la EI en sus inicios se distanció de una sociología institucionalizada objetivizante, que responde a una cultura patriarcal y se presenta a sí misma como universal, excluyendo a las mujeres en los textos, en el habla y en el poder (Smith, 1987). En coherencia con las críticas feministas, Smith propone, en contraste, una sociología para las mujeres centrada en el punto de vista de ellas y en las realidades de sus vidas cotidianas, encorporadas y situadas para explorar, a partir de esas experiencias, las maneras en que la sociedad las moldea y organiza (Smith, 2006, 2005, 1997, 1992, 1990 y 1987). Posteriormente, el carácter feminista de la EI trasciende como estrategia de investigación para el conjunto de otros colectivos de marginalizados (O’Neill, 1998).

El objetivo de Smith es proponer un proyecto sociológico que "comienza en las realidades de la experiencia de las mujeres" (Smith, 1992, p. 88), situándose en su experiencia -ella incluida- 
como mujeres. Militante del movimiento de mujeres (Campbell, 2003), aprendió a explorar su experiencia de opresión y a situarse desde ese punto de vista, lo que transformó diversos aspectos de su vidas, como las maneras de pensar, trabajar y relacionarse. Estas experiencias y descubrimientos dieron origen a un giro epistemológico, ontológico y conceptual que, si bien en un principio no tenía nominación, Harding (1986), aglomerando distintas perspectivas, formalizó posteriormente como el standpoint o punto de vista. Esta perspectiva, que comienza desde la experiencia de las mujeres, es distinta de un conocimiento feminista (Smith, 1997), ya que el standpoint como estrategia de investigación puede ser operacionalizado por diversos grupos marginalizados (O'Neill, 1998) y, tras las experiencias situadas, busca rastrear las relaciones de control.

La Sociología clásica, a diferencia del standpoint, separa el conocedor de lo conocido, extirpando lo situado del conocimiento y deslocalizándolo. Por su parte, el standpoint de las mujeres vuelve hacia las realidades de sus vidas, del modo en que ellas las viven en sus particularidades y mundos cotidianos, apuntando a la idea de "Usted está aquí" (Smith, 1997, 1992). Lo que hace el standpoint es desplegar discursos, conceptos y teorías en las realidades, como prácticas reales de las personas que las viven y experimentan.

Por lo tanto, el punto de partida de la EI es una experiencia constitutiva, que se refiere al hecho de localizarse corporalmente en y desde el punto de vista de las mujeres, en tanto invisibilizadas, dominadas, y marginadas. "La investigación no comienza al interior de la organización conceptual o las relevancias [relevances] del discurso sociológico, sino en la experiencia real en tanto incrustada en las formas históricas particulares de las relaciones sociales que determinan esa experiencia" (Smith, 1987, p. 49). Si bien es cercano, este punto de partida no se puede confundir con el conocimiento situado de Haraway (1988), ya que mientras este último considera la objetividad como un conocimiento situado, es decir, que "sólo una perspectiva parcial promete una visión 
objetiva" (Haraway, 1988, p. 583), la EI parte de las experiencias cotidianas, que son localizadas y encorporadas, para luego comenzar a rastrear las relaciones de control sobre esas experiencias que están coordinadas desde lejos y de modo translocal.

En este sentido, uno de los cambios fundamentales de tomar el standpoint es considerar la vida cotidiana como problemática (Smith, 1987). Implica cambiar, desde una manera de conocer objetivizante o ideológica, muy asentada en la sociología clásica, a una manera experiencial de conocer; constituye el giro epistemológico fundamental de esta perspectiva (Deveau, 2008).

Este giro no privilegia a algún conocedor en particular, sino más bien lo que hace es:

... cambiar el terreno del conocimiento, el lugar donde comienza la investigación. (...) El punto de vista de las mujeres propone un point d'appui diferente: comienza un paso antes del giro cartesiano que olvida al cuerpo. El cuerpo no está olvidado; por lo tanto, el sitio real del cuerpo no está olvidado. La investigación comienza con el conocedor que está en efecto localizado (Smith, 1992, p. 91).

El objetivo de la investigación EI es explorar, a partir de las experiencias cotidianas, diurnas y nocturnas de los sujetos conocedores, qué es lo que va más allá de esas experiencias, las relaciones sociales, la organización y las relaciones de control que emergen de los complejos institucionales que coordinan y administran dichas experiencias. Es decir, la investigación se centra en localizar las experiencias cotidianas de opresión en los contextos que producen dichas relaciones y experiencias, explorando cómo penetran en el mundo cotidiano, pero permanecen invisibles en él (Rankin, 2017; Deveau, 2008; Grahame, 1998; Smith, 1997, 1992).

Según O’Neill, “una mejor comprensión de la realidad puede ser alcanzada llevando a cabo investigación desde las localizacio- 
nes sociales [social locations] dåe los grupos marginalizados, que desde las posiciones de los grupos dominantes" (O'Neill, 1998, pp. 130 y 131). Esta estrategia permitiría localizar y mapear las fuerzas que operan desde lejos y que expresan, en lo local, relaciones de control translocales, haciendo visibles conexiones invisibles y haciendo posible la comprensión de las implicaciones más amplias de las experiencias localizadas (Rankin, 2017; Deveau, 2008). La EI busca resituar las agencias que se transmiten desde instituciones en los cuerpos y en las experiencias mismas de las personas.

La EI despliega una serie de conceptos para conformar su marco de investigación. A continuación, revisamos algunos de ellos:

Prácticas cotidianas, organización social y relaciones sociales. Las prácticas cotidianas son actividades de coordinación (Smith, 1992) que pueden ser intercambios entre dos prácticas en las que las personas se conectan, como comprar-vender, y que son visibles y empíricas. Pero tras esas relaciones visibles se esconde una organización social que es invisible y que es lo que se intenta visibilizar y explicar. De este modo, lo que se busca es dilucidar las relaciones sociales translocales implicadas en dicha relación social (Rankin, 2017; Deveau, 2008).

Deveau (2008) ilustra esto con la práctica de comprar carne en el supermercado. Lo que se observa ahí es la compra-venta, pero lo que interesa observar es el proceso por medio del cual esa carne llega a la tienda, que permanece invisible. La EI buscaría develar a los productores, la crianza, las normativas estatales, el matadero, el transporte y las condiciones del supermercado, para que la persona pueda comprar la carne, entre otras. En las actividades ordinarias diarias están implicadas un sinnúmero de relaciones sociales que no son observables en los escenarios locales y que son representadas por personas, "a menudo sin su conocimiento consciente" (Campbell y Gregor, 2002, p. 31). En estos términos, las relaciones translocales implicadas en las relaciones 
sociales cotidianas se develan de una forma similar a como lo plantea la etnografía multisituada, siguiendo la movilidad de los objetos que median la relación, pero, en el caso de la EI, con un énfasis en las plataformas institucionales que hacen posible esa movilidad.

La problemática de investigación. Ésta aparece en la brecha entre el conocimiento abstracto de una situación y el conocimiento de las personas. La problemática no es una pregunta de investigación, ni tampoco un problema que el informante pueda expresar, sino más bien se enfoca en descubrir "cómo las experiencias cotidianas están enganchadas en, y coordinadas por, las relaciones de control" (Deveau, 2008, p. 12). Es fundamental centrarse en las maneras en que las relaciones de control translocales, coordinadas, afectan las experiencias locales de los sujetos con quienes se está trabajando.

La problemática emerge de la cotidianidad del punto de vista de los informantes. Esta puede ser descrita, incluso, usando una sola entrevista clave, "pero lo que importa es cómo las descripciones de los quehaceres de las personas proveen destellos de las relaciones de control que están siendo activadas -aquellas relaciones que tienen implicancias más amplias" (Rankin, 2017b, p. 8). Se trata entonces de develar las relaciones de control.

Relaciones de control. Son relaciones sociales que se generan alejadas de la experiencia y del standpoint, pero que son activadas en los escenarios locales. Ellas moldean la manera en que las acciones con sentido son llevadas a cabo y cómo son representadas. Ellas coordinan "lo que las personas conocen sobre lo que está sucediendo -incluso si ese conocimiento no coincide con lo que se conoce por estar ahí" (Rankin, 2017, p. 3). Este término apunta a dilucidar las conexiones entre diferentes instituciones (Estado, cuerpos profesionales, corporaciones y academia, entre otros) que, mediadas por textos, organizan y regulan la sociedad (Bisaillon, 2012). De este modo, generan conceptos especializados, lenguajes técnicos y categorías que moldean lo que se co- 
noce del mundo, coordinando las experiencias situadas de las personas (Smith, 1992).

Estas relaciones de control "se definen como escenarios [venues] textuales (tales como la legislación, paneles de expertos, hacedores de política, acciones de administración y gestión), donde el poder es generado y perpetuado en la sociedad a través de múltiples sitios" (Wright, 2003, p. 244). La construcción de esos textos es una operación de abstracción en la que no hay lugar para la experiencia vivida, ya que ella, desde una perspectiva institucional, no tiene relevancia en la construcción -legal, oficial- de ningún evento significativo o cotidiano.

Una declaración oficial textualizada, tal como puede ser un plan de ordenamiento territorial, es entendido por la EI como la construcción de una explicación o un informe ideológico, en el que se insertan marcos conceptuales formales - de control- y se suprimen las experiencias de los sujetos respecto de ese evento real y vivido por ellos, a pesar de que el informe dice dar cuenta de ese evento. Las relaciones de control son, por lo tanto, las prácticas que activan, a través de textos, determinado modo en que las cosas suceden, organizan el trabajo distante de las experiencias, pero son activadas por personas que se encuentran en la localización. Por lo tanto, son:

... actividades de regulación [governing] que dependen de la selección, categorización y/o de la objetivización de aspectos del mundo social a fin de desarrollar hechos y conocimiento sobre el que tomar decisiones. Las relaciones de control activan prácticas de conocimiento que subsumen cómo un problema es conocido y experimentado desde una localización situada [standpoint location]. (Rankin, 2017, p. 33)

Textos y mediación de textos. Los formatos textuales, cuyas configuraciones van desde documentos, reportes, permisos, formularios, hasta dibujos, videos y registros de audio, se constituyen como centrales en tanto formas de conocimiento y de or- 
ganización discursiva que moldean las vidas cotidianas de las personas. Es decir, como instrumentos de control que son replicados y que circulan por diferentes localizaciones. Entendidos como "los componentes translocales de la organización social cotidiana" (Campbell, 2014, p. 1495), los textos son materialidades que traen a la realidad localizada, determinadas maneras de dar sentido a las prácticas y que pueden ser leídas en varios escenarios diferentes (Smith, 1992). Son los formatos textuales los que son constitutivos del proceso de control en las sociedades contemporáneas (Grahame, 1998, p. 349), porque funcionan al interior de una inscripción institucional translocal que tiene efectos en lo local, ya que organizan, regulan, y coordinan las relaciones sociales en diferentes localizaciones.

Los textos limitan las posibles comprensiones de lo que se lee, imponiendo una comprensión homogénea de lo que es leído por una variedad de personas, en distintos sitios, que los utilizan de modo rutinario. También tienen la capacidad de coordinar las acciones de personas en distintos lugares, incluso si no se conocen, por los requerimientos existentes para trabajar con ellos (Campbell y Gregor, 2002). De este modo, los análisis textuales pueden servir para destapar las prácticas ideológicas que producen el tipo de conocimiento basado en las relaciones de control (Deveau, 2008). Como se verá en detalle más adelante, los reglamentos, por ejemplo, aparecen en forma de textos accesibles a los aparatos que elaboran informes de situaciones (equipos técnicos en terreno, por ejemplo), que reflejan un marco regulatorio ideológico (leyes y políticas públicas), pero no reflejan el punto de vista o la experiencia de los sujetos sobre los que se informa, generando una separación entre las experiencias reales y lo que representa institucionalmente la situación, en tanto dimensión del poder institucional, ejerciendo poder "cuando son activados por los miembros del aparato de control" (Deveau, 2008 , p. 10). Por lo tanto, más allá de las intenciones declaradas, estos textos con dificultad podrían dar cuenta de las realidades que se viven. 
Complejo institucional. Para la EI, las experiencias son moldeadas por procesos institucionales que están ubicados lejos de ellas, lo que hemos llamado "relaciones translocales". En el proceso de investigación, la EI busca seguir la pista de esos procesos institucionales que regulan y coordinan las prácticas de las personas en escenarios locales (Rankin, 2017b). Pero la EI opera con un concepto de institución diferente a lo que comúnmente es entendido por tal. Más que una organización singular, la institución es más bien un régimen, complejos procesos funcionales en los que una variedad de formas de organización están entretejidas (Grahame, 1998) o, como dice Deveau (2008b , pp. 110 y 111), un "vasto despliegue de procesos de trabajo coordinado y cursos de acción teniendo lugar en múltiples locaciones". Por ello, más que centrarse en realizar descripciones del funcionamiento de organizaciones singulares, la EI busca, partiendo de la experiencia e identificando y recurriendo a los textos, dar cuenta de cómo el trabajo de las personas y sus acciones con sentido están articuladas con otras acciones, coordinadas a través de las relaciones de control, en y desde sitios alejados.

En resumen, para la EI el/la conocedor/a está situado/a en las realidades de su vida, y su interés en lo social busca dilucidar las actividades de coordinación y concertación que operan más allá de su experiencia. Para la EI la textualidad es fundamental, porque es el puente entre lo real y la explicación que se hace de ello, siendo -los textos- componentes materiales de la organización social (Campbell, 2014), que traen a contextos de lectura reales formas de significación fijas que pueden ser abstraídas y leídas en otras localizaciones, translocalizando de esta forma su significación. Estas son formas en las que el poder es generado y sostenido, ya que las "funciones de organización y control están crecientemente establecidas en formas de organización y de relaciones distintas, especializadas y (hasta cierto punto) autónomas mediadas por textos" (Smith, 1992, p. 93). 


\section{Principios procedimentales de una EI}

No existe un modo estandarizado de realizar investigación en EI, ya que busca descubrir, más allá de cualquier experiencia individual, "cómo las actividades de la gente están coordinadas" (Smith, 2006, p. 1), los investigadores siguen ciertos principios procedimentales. El más general de ellos, es que la EI se basa en la experiencia cotidiana, en que "el análisis comienza en la experiencia y vuelve a ella, habiendo explicado cómo la experiencia sucedió de la manera en que lo hizo" (Campbell, 1998, p. 56).

El desafío es concebir las experiencias cotidianas de las personas como localizaciones de una problemática, entendida como un conjunto de preguntas teóricas y de conceptos que están relacionados (Grahame, 1998). Desde ahí, se observan cómo esas experiencias cotidianas están enganchadas y coordinadas por relaciones de control (Deveau, 2008). El/la etnógrafo/a institucional busca identificar cómo en la vida cotidiana el conocimiento está organizado y cómo este descansa en conceptos objetivados y separados de las experiencias de personas situadas. Justamente, ese conocimiento está inserto en las relaciones de control y en las prácticas discursivas de su producción (Rankin, 2017a).

Operativamente, la EI trabaja con dos niveles de datos. En primer lugar, los datos de entrada [entry level data], que son las problemáticas que surgen a partir del examen de las interacciones significativas y subjetividades de los sujetos en el habitar cotidiano y en sus localizaciones particulares (Deveau, 2008 y 2008b; Wright y Rocco, 2007; Campbell y Gregor, 2002; O'Neill, 1998). En este momento se puede observar empíricamente y dar cuenta del objeto de estudio que es la vida cotidiana, localizada espacial, temporal y corporalmente en tanto problemática. Los datos primarios, recogidos de las experiencias cotidianas, se obtienen desde los escenarios locales y respecto de los individuos que interactúan en ellos. 
El segundo nivel se compone de los datos translocales [translocal data], que se extienden más allá de la experiencia de los sujetos localizados (el primer nivel de datos), hacia las relaciones sociales (Wright y Rocco, 2007) y, por ende, relaciones de control. El propósito de recolectar y producir datos en este nivel es comprender las relaciones, similares o análogas, que pueden tener las personas en otras localizaciones respecto de las encontradas en el primer nivel de datos, haciendo posible de explicar las relaciones de control (Deveau, 2009) que son por definición translocales. Aquí los textos devienen centrales pues, al ser materialidades susceptibles de ser leídas en diversos escenarios de modo estandarizado, imponen relaciones de control en una extensión translocal. La recolección en este segundo nivel permitiría explicar la manera en que los textos coordinan las experiencias en diferentes localizaciones y también explicar esas relaciones de control (Campbell, 2014; Deveau, 2009; Wright y Rocco, 2007).

Estas fases dan cuenta del principio de comenzar por la experiencia y terminar con ella, explicando en un proceso iterativo cómo esa experiencia sucede de la manera en que lo hace. Esto se puede realizar mediante mapeos, reportes, diagramas o indexaciones, que localizan las fuerzas que operan de modo translocal y que regulan las prácticas locales a través de las relaciones de control (Rankin, 2017b). Haciendo referencia a la metáfora del “Usted está aquí" de los mapas de los malls (Smith, 1992), y usando diferentes herramientas para revelar lo que se da por sentado (Grahame, 1998; Smith, 1997), se puede producir y usar cartografías sociales para comprender y desafiar los conocimientos producidos abstracta y extralocalmente en la transformación de las experiencias (Rankin, 2017b; Deveau, 2008; Campbell y Gregor, 2002).

\section{Implementaciones desde la perspectiva de EI}

Se puede encontrar una amplia variedad de investigaciones que utilizan la EI como estrategia, cuyos alcances y campos de aplicación se amplían continuamente. Si bien parecen fragmen- 
tados, "nos enseñan más y más sobre la organización compleja y entrelazada de las relaciones de control, y más y más sobre cómo los procesos institucionales están coordinados y 'operan'” (Smith, 1992, p. 97).

En ese abanico interdisciplinar destacan, en particular, los temas de salud, educación y crianza, como la organización textualizada de servicios de enfermería (Rankin y Campbell, 2006 y 2009; Campbell, 1984), las políticas de rehabilitación, las reformas de gerencia pública y sus impactos en las personas en procesos de rehabilitación (Paulsen, 2015); la integración entre la asistencia y los cuidados médicos y la educación para niños/as con condiciones especiales de salud o discapacidad ( $\mathrm{Ng}$ et al., 2013); la ideología de la maternidad monoparental y su relación con la escolaridad (Griffith y Smith 2004; Grahame, 1998; Griffith, 1995); la educación universitaria de trabajo social con respecto al standpoint de las personas homosexuales (O’Neill, 1998), y las personas en situación de discapacidad, respecto del lugar de trabajo en el sistema público (Deveau, 2008b).

Sin embargo, a pesar de la capacidad demostrativa y explicativa de esta perspectiva, en América Latina la EI ha tenido aún un impacto limitado. Si bien se puede encontrar algunas investigaciones latinoamericanas basadas en EI, en ocasiones se confunde con otras estrategias metodológicas, en particular con la que podría denominarse "Etnografía de las instituciones", cuya lógica se centra más bien en etnografiar los efectos que instituciones singulares tienen sobre las experiencias de los sujetos objeto de ellas (i. e. estudiantes en la escuela, políticas universales y programas focalizados, seguridad alimentaria), más que desde el habitar situado de los sujetos, como son los trabajos de Vázquez (2017), Torres et al. (2015), Vizcarra y Farfán (2012) y Hevia (2011).

Entre los trabajos destacados que utilizan la EI en América Latina se puede encontrar el estudio de los trastornos mentales y su medicalización en Brasil (Medeiros y Álvarez, 2016); el sistema público de educación musical desde una perspectiva poscolo- 
nial (Rosabal-Coto, 2016) o las experiencias contradictorias de la lactancia materna y la maternidad como experiencia e institución (Yáñez, 2010). Además, se puede hallar un incipiente desarrollo de esta perspectiva en tesis de maestría y doctorado, como el estudio de las prácticas institucionales dominicanas para la protección de derechos de niñas, niños y adolescentes (Nivar e Hiciano, 2017), y el análisis de los derechos sexuales y reproductivos en la formación de pregrado de médicos colombianos (Restrepo, 2014).

En la siguiente sección se explora el modo en que la EI es susceptible de aplicarse a los estudios del habitar urbano, para demostrar cómo, partiendo de la experiencia situada, se puede identificar una problemática rastreando en primera instancia relaciones de control que operan translocalmente. En el marco de la investigación en que se basa este trabajo, la EI resulta ser una estrategia de gran potencial, ya que el objetivo de trabajo es comprender la producción de conocimientos que surgen a partir de la interacción entre prácticas de intervención urbana y prácticas cotidianas de habitar que dan forma a territorios en Santiago de Chile. Esto implica comprender cómo los saberes cotidianos del habitar socioespacial muchas veces entran en conflicto con las relaciones de control que intenten las diversas formas de intervención urbana. El proyecto busca detectar las dislocaciones y de conocimientos y saberes en diferentes tipos de intervenciones, expresadas en sistemas de transporte, programas de recuperación de barrios y programas culturales barriales, entre otros.

Para este artículo nos centraremos en el caso del Programa de Recuperación de Barrios "Quiero Mi Barrio" (MINVU), que trata de problematizar respecto de los conocimientos y saberes que median entre quienes habitan en un barrio ubicado en la ciudad de Santiago y la intervención misma, así como el régimen institucional que rodea dicha intervención. El trabajo de campo de este estudio aún se encuentra en ejecución, por ello se presentan ejemplos de la primera fase de la EI: la del develamiento de una problemática. La información presentada está elaborada bajo una metodología etnográfica colectiva (Jirón e Imilán, 2016) por un 
equipo compuesto por cinco etnógrafos participantes en la investigación y el equipo de investigadores del proyecto, en un trabajo realizado a partir de marzo de 2018 y cuyo proceso terminará a inicios de 2019.

Tal como explican Jirón e Imilán (2016), la investigación urbana, y particularmente la investigación sobre habitar urbano contemporáneo, posee altos niveles de complejidad, dinamismo y diversidad, que requiere de estrategias metodológicas que integren disciplinas y estrategias. La adopción de un enfoque etnográfico precisa de una adaptación de su aplicación tradicional, permitiendo la realización de un trabajo dinámico y colectivo, así como la posibilidad de incorporar el trabajo interdisciplinario a la producción y análisis. Con este propósito, la etnografía colectiva otorga la posibilidad de desarrollar un trabajo profundo, a partir de trabajo de campo breve en el tiempo pero intenso en su producción. Es decir, planteamos la posibilidad de construir etnografías mediante el trabajo articulado, en un equipo de investigadores que observan variables similares y comparten de forma intensa sus propias experiencias etnográficas. La práctica etnográfica es una de tipo "encorporado", porque esta etnografía se hace con el cuerpo. En nuestro caso, "encorporar" los conceptos y metodologías planteados en el proyecto es fundamental para lograr empatía en el etnógrafo. Junto con el aprendizaje corporal de la práctica etnográfica, el análisis y debate multidisciplinario, que reorienta registro y representación, completan nuestra formulación de etnografía colectiva. A partir del conjunto de sesiones grupales y talleres se ensambla interdisciplinariamente el ejercicio individual de investigación.

La metodología de etnografía colectiva ha sido utilizada por el equipo de investigación en diversas oportunidades (Jirón, Imilán e Iturra, 2016; Jirón e Imilán, 2015) con el fin de conocer mejor formas de habitar en algunas ciudades del país (Santiago, Concepción, Alto Hospicio y Padre Las Casas). Sobre la base de esta experiencia, se ha producido un modelo metodológico que organiza de manera colectiva el trabajo de campo en cinco momentos. 
El primer paso involucra la conformación del equipo de etnógrafos junto con su capacitación y acercamiento a formas de observación de saberes del habitar, intentando buscar formas en las que estos saberes se relacionan o no con los saberes de las intervenciones. La segunda consiste en el proceso de selección de los casos, más el proceso de diseño y aplicación de instrumentos. De acuerdo con el carácter multisituado de las prácticas de habitar, en las que los espacios que se habitan no están limitados a una sola locación, sino que se vinculan entre sí a modo de trayecto, el trabajo etnográfico implica observar un número acotado de hogares cuyos miembros realicen prácticas de habitar con relación a los programas de intervención. La tercera etapa realizará una etnografía espacio temporal del habitar, basada en un análisis de interacciones significativas en el territorio (Jirón e Imilán, 2016), integrando las relaciones que emergen entre el cuerpo, territorio, espacialidad, alteridades, objetos, significados, racionalidades y estrategias espaciales. La cuarta, involucra la producción de relatos etnográficos, mientras que la última etapa se concentra en el análisis interdisciplinario de estos datos, tendientes a la problematización que implica la EI.

Las ventajas de este tipo de visión colaborativa de la etnografía implican profundizar las relaciones entre los etnógrafos, los miembros del equipo de investigación, las personas que participan en los estudios y aquellos que otorgan apoyo e información para el trabajo de campo. A continuación se presenta una parte del trabajo colaborativo en curso, en el que se pueden detectar formas de problematizar los saberes y conocimientos vinculados al habitar y a las intervenciones urbanas.

Intervención del Programa de Recuperación de Barrios "Quiero Mi Barrio" y un barrio popular de Santiago. Primeros pasos para una Etnografía Institucional

En un barrio popular en la zona centro sur de la ciudad de Santiago, el año 2014 comienza el proceso de intervención social y urbana del Programa "Quiero Mi Barrio" (en adelante, PQMB), 
perteneciente al Ministerio de Vivienda y Urbanismo (MINVU), cuyo objetivo es "contribuir al mejoramiento de la calidad de vida de los habitantes de barrio que presentan problemas de deterioro urbano, segregación y vulnerabilidad social, a través de un proceso participativo de recuperación de los espacios públicos y de los entornos urbanos de las familias", según consigna el Decreto Supremo actualizado $N^{\circ} 14$, de 2007, que reglamenta el Programa de Recuperación de Barrios.

Este programa llamó a un polígono mencionado como "Barrio José ${ }^{7 \prime \prime}$, conformado por las poblaciones El Huaso, Berlín y José Andrés ${ }^{8}$, colindantes unas con otras, pero que no mantenían una relación funcional entre ellas, previo a la intervención.

La selección y postulación conjunta de dichas poblaciones al programa fue gestionada por la municipalidad, aunque se desconoce la razón por la cual se agruparon las tres, en lugar de postular polígonos más acotados. No obstante, previo a la postulación, los vecinos de El Huaso habían propuesto a la Secretaría Regional Ministerial de Vivienda y Urbanismo (SEREMI MINVU) arreglar la cancha de fútbol de su población. Al respecto, un integrante del Equipo de Barrio del Programa, comenta sobre el proceso de gestión:

“... fue aleatorio, porque generalmente los barrios es una población y hay una identidad en común. En este caso, para armar el polígono se juntaron las tres poblaciones. Y en esto hubo ganadores y perjudicados, porque obviamente la cancha El Huaso es una intervención gigante".

Otra integrante del mismo equipo también comenta que el alcalde presentó a los vecinos, en julio de 2014, lo que iba a ser la intervención de la cancha de la población El Huaso y el PQMB llegó en noviembre de ese año, lo que indica que los planes de la 
cancha estuvieron presentes desde antes de la intervención. En el mismo sentido, el Coordinador Territorial de la SEREMI ${ }^{9}$ menciona que "El Huaso obviamente tiene un cuento bien visible, en donde hay muchos intereses. Están todos los ojos metidos ahí, que en otros barrios no pasa mucho".

Otro hito territorial de la intervención del PQMB es la Plaza José Andrés, perteneciente a la población del mismo nombre. En este caso, su intervención urbana material ha sido más compleja, debido a que en ella se encuentra un árbol/animita pintado de colores y con luces incrustadas, que algunas noches se encienden en honor a un narcotraficante fallecido de forma violenta, años atrás. Este espacio se constituyó como un lugar significativo para algunos de los vecinos de la población y ha sido fuente de ciertos conflictos debido a que la intervención del PQMB requería la remoción del dicho árbol/animita.

Estos dos ejemplos del proceso de intervención del PQMB, la cancha El Huaso y la Plaza José Andrés, pueden constituir lo que más arriba se describió como los datos de entrada y son ilustrativos de cómo un acercamiento basado en la Etnografía Institucional puede servir para, por un lado, identificar un dislocamiento entre la experiencia cotidiana diurna/nocturna de las personas y su alteración o abstracción en otra cosa, y, por otro, para esbozar la direccionalidad de un posible mapeo respecto de las relaciones de control translocales que afectan las experiencias cotidianas situadas.

En el primer caso, se trata de dilucidar lo que Smith (2006 y 1987) denomina una "problemática", entendida como una separación entre la experiencia y su abstracción en algo distinto. Se trata del punto crítico, en que un individuo se da cuenta que lo que está sucediendo está siendo traducido en otra cosa, sin considerar la experiencia de quienes habitan el lugar intervenido. En

Secretaría Regional Ministerial es la entidad técnica del Ministerio de Vivienda y Urbanismo encargada de apoyar la ejecución del PQMB a nivel de la Región. 
el caso de la Plaza José Andrés es relativamente fácil identificar que el árbol/animita es significativo para los vecinos que ocupaban la plaza antes de la intervención del PQMB. A este se le cuelgan y prenden luces, se reúnen a su lado o, incluso, se rechaza precisamente por lo que evoca. El árbol/animita contiene todas las características que, según Cresswell (2004), debiese tener un lugar: espacialidad, ubicación y significado. Dicho árbol/animita es, por tanto, constitutivo y significativo de las experiencias cotidianas en tanto vecinos de la Plaza José Andrés, independientemente de la valoración que se tenga del narcotraficante por el que dicha animita se erige.

El hecho de que el PQMB arribe en 2014 y, al intervenir la Plaza José Andrés, requiera la remoción del árbol/animita, habla del desajuste que existe entre la experiencia de las personas que habitan el lugar cotidianamente y los interventores que, para lograr cumplir unos objetivos homogéneos y deslocalizados, remueven y transforman un lugar identitario, relacional e histórico.

El caso de la intervención de la cancha de la población El Huaso puede ser ilustrativa de cómo están coordinadas determinadas relaciones de control que afectan las experiencias cotidianas, para comenzar a mapear los diversos esquemas, formas y grados en que opera el control translocal del régimen institucional, a partir del análisis de textos.

Como se mencionó, la cancha El Huaso es relevante, porque, como dijo el coordinador territorial de la SEREMI, en torno a esta hay intereses que la hacen visible. Se trata de una serie de clubes deportivos y de otros usos menos institucionalizados y regulares, que transforman a la cancha en un espacio significativo de esparcimiento para los habitantes cercanos. Sin embargo, ni en la planificación ni en la gestión, ni tampoco en la posterior ejecución de dicha intervención participaron los vecinos. En las conversaciones con los habitantes del lugar aparece la idea de que el proceso fue confuso, llevado a cabo por la municipalidad, aprovechando las buenas relaciones que tenían con la administración guberna- 
mental. Esto cobra sentido cuando se observa también que en la selección del polígono a intervenir tampoco participaron los vecinos, a pesar de que el objetivo primero del PQMB era mejorar las condiciones de vida "a través de un proceso participativo de recuperación de los espacios públicos y de los entornos urbanos de las familias". De ahí aparece, en el mismo sentido que la Plaza José Andrés, la problemática de dislocamiento entre la experiencia y su abstracción en algo distinto. Lo particular, en el caso de la cancha de El Huaso, es que permite dar cuenta de la participación de otros actores externos en la constitución, coordinación y control de las experiencias de quienes habitan el espacio: la municipalidad, el PQMB, el ministerio y el gobierno, ausentándose de este proceso los vecinos de las poblaciones José Andrés, Berlín y, en menor medida, algunos de los habitantes de la población El Huaso, beneficiaria directa de la intervención.

La problemática surgida a partir de la cancha de El Huaso permite dar pie a un seguimiento cuyo objetivo sea dilucidar cuál es el régimen institucional que está comprendido en la coordinación y producción de la experiencia situada de los habitantes de las tres poblaciones, además de explorar la posibilidad de que la coordinación y producción de dichas experiencias se realicen de modo translocal, buscando otras experiencias situadas que sean equivalentes. Esto correspondería al segundo nivel de datos en el procedimiento de una EI, o lo que Wright y Rocco (2007) llaman "datos translocales", que se extienden más allá de la experiencia, hacia la identificación de las relaciones de control y coordinación que hacen que esas experiencias translocales puedan ser análogas o equivalentes. En este segundo nivel, los textos devienen relevantes, porque al ser susceptibles de ser leídos y comprendidos de la misma manera en diferentes escenarios, ellos impondrán relaciones de control translocal y forman parte de la segunda fase de la EI que este proyecto busca dilucidar, a partir de las problemáticas detectadas.

Desde una mirada de la EI, en este caso los textos podrían referirse, por ejemplo, al Manual de Procedimiento del Progra- 
ma de Recuperación de Barrios aprobado el 2008 y modificado en 2009 y 2011, y dar cuenta que en este se plantea -contra los objetivos del programa- que la selección de barrios la realiza el MINVU, de acuerdo con indicadores de deterioro urbano y vulnerabilidad social, categorizando barrios según complejidad media o alta, y en relación a la cantidad de viviendas (MINVU, 2011, p. 4). Además, la estrategia de intervención para todos los barrios se compone de tres fases: elaboración de un Contrato de Barrio, llevar a cabo un Plan Maestro, Plan de Gestión de Obras y Plan de Gestión Social, y un cierre con evaluaciones, sistematizaciones y agenda futura (MINVU, 2011, pp. 8 y 9).

Estas definiciones y procedimientos estandarizados del PQMB pueden dar pie a comenzar a mapear las maneras en que se producen las definiciones conceptuales, en que se elaboran, diseñan, implementan y evalúan las políticas públicas de barrios e incluso del ministerio, así como las discusiones académicas en torno al concepto de "barrio", entre otros elementos que permitirían identificar el régimen institucional involucrado en el proceso de intervención, coordinación y control sobre la experiencia de los sujetos que habitan la ciudad y, en particular, los barrios definidos y seleccionados por el PQMB. También, y complementariamente, pueden dar pie a investigar cómo experiencias equivalentes pueden estar coordinadas mediante el PQMB de modo translocal, a través de estas mismas definiciones y procedimientos textualizados y estandarizados en otros escenarios, explorando cualquiera de los 520 barrios intervenidos por el programa hasta el momento en el país.

\section{Reflexiones y desafíos para una Etnografía Institucional en el campo de lo urbano}

En las páginas precedentes se describieron los principales elementos que componen la Etnografía Institucional como estrategia de investigación de la producción de conocimientos desde una mirada situada, tanto en su aspecto teórico como en diversos 
campos de estudio empírico de investigación. También se esbozó una manera de poner en práctica esta estrategia en estudios del habitar urbano, por medio de información recabada en el marco del proyecto de investigación en curso.

El objetivo ha sido presentar la EI como estrategia investigativa que, partiendo de la experiencia, puede mapear los procesos por los que esa experiencia puede ser coordinada por un régimen institucional que genera relaciones de control activadas en diferentes escenarios, y que la traducen en algo distinto, ajeno a las significaciones de los sujetos, en sus vidas cotidianas.

En esta aproximación al campo de estudio del habitar urbano, la EI demuestra ser útil y pertinente para comprender la manera en que esos conocimientos y experiencias se producen por esa articulación entre la práctica cotidiana del habitar situado de sujetos que dan forma y significaciones al territorio y las prácticas de intervención urbana externas que, sobre ese territorio, modelan esas experiencias estableciendo relaciones de control por medio de textos. No se trata de verificar si los supuestos principios de participación que proponen programas de mejoramiento de barrios como el PQMB se cumplen y en qué grados, lo que supondría la evaluación de la implementación de dicho programa mediante la verificación del nivel de éxito o fracaso de sus propios indicadores. La EI apunta, por el contrario, a develar las relaciones de control que produce el PQMB y, de esta manera, identificar los distintos conocimientos y saberes en juego que producen una dislocación entre la práctica cotidiana de los habitantes y las intervenciones en el territorio y probablemente en las prácticas de planificación urbana.

La definición de "barrio", como experiencia identitaria fuertemente situada, se ve tensionada por los procedimientos de delimitación amparados en una serie de procedimientos, semánticas y prácticas concretas de los agentes de la intervención que se expresan en diversos textos. Asimismo, los usos locales de un espacio abierto como es la cancha El Huaso son invisibilizados y des- 
conocidos por los interventores, quienes no saben los procesos de coordinación entre los diferentes actores que han dado ocupación a la cancha, antes de su intervención con inversión pública para su "mejoramiento". Queda por indagar si esas experiencias modeladas por el régimen institucional, modelan también experiencias locales situadas en escenarios distintos al estudiado, lo que constituye la siguiente etapa de la investigación en curso en otros territorios de la ciudad de Santiago.

Se puede decir que, así como en los campos de estudios de la crianza, la salud y la educación, la EI puede ser una estrategia adecuada y fructífera para dilucidar cómo las experiencias locales se coordinan extralocalmente también en los estudios del habitar y lo urbano, o más específicamente con el urbanismo y la planificación urbana. Sin embargo, existe una variedad de campos en los que la EI no se ha sido aún suficientemente explorada. Entre ellos, los estudios urbanos vinculados a aspectos de desigualdad urbana, exclusión social y efectos en el espacio vinculados con intervenciones de políticas públicas, entre otros, en los que la EI eventualmente podría aportar nuevas maneras de observación y comprensión. Precisamente, este proyecto buscar llenar ese vacío de implementación metodológica combinando estrategias etnográficas del habitar cotidiano, a partir de estrategias colaborativas, junto con esta mirada de la EI más vinculada a los conocimientos y detrás de las intervenciones, que es lo que se busca indagar.

Por último, esta estrategia ha sido escasamente desarrollada en América Latina y Chile, pero, tal como se presentó, podría abrir nuevos campos de debates al tensionar las formas de conocimiento que guían el actuar cotidiano de los habitantes y las formas en que las políticas públicas intentan controlarlo.

\section{Referencias}

Acuña, D., Lara, B., Basaure, C., Navea, O., Kripper, C. y Saldías, F. (2015). Estratificación de Riesgo (Triage) en el Servicio de Urgencia. Revista Chilena de Medicina Intensiva, 3(2), 79-86. 
Auyero, J. (2012). Los sinuosos caminos de la etnografía política. Revista Pléyade, 10, 15-36

Billo, E. \& Mountz, A. (2016). For institutional ethnography: Geographical approaches to institutions and the everyday. Progress in Human Geography, 40(2), 199-220.

Bisaillon, L. (2012). An Analytic glossary to social inquiry using institutional and political activist ethnography. International journal of Qualitative Methods, 11(5), 607-627.

Blanco, M. (2012). Autoetnografía: una forma narrativa de generación de conocimientos. Andamios, 19(19), 49-74.

Campbell, M. (1998), Institutional Ethnography and Experience as Data. Qualitative Sociology, 21(1), 55-73.

Campbell, M. (2003), Dorothy Smith and knowing the world we live in. The Journal of Sociology $\mathcal{E}$ Social Welfare, 30(1), Special issue on Institutional Ethnography: Theory and Practice, 3-22.

Campbell, M. (2014), Institutional Ethnography (IE), Texts and the Materiality of the Social, 47th Hawaii international conference on system science, 2014, 1495-1504.

Campbell, M. \& Gregor, F. (2002). Mapping social relations: A primer in doing institutional ethnography. Toronto: Garamond Press.

Cresswell, T. (2004). Place: A Short Introduction. Wiley-Blackwell.

Deveau, J. L. (2008). Examining the Institutional Ethnographer's Toolkit. Socialist Studies / Études Socialistes, 1-20

Deveau, J. L. (2008b). Workplace accommodation for disabled workers in the Canadian federal public service: a textually mediated social organization (Ph. D. Dissertation). University of New Brunswick, Canada.

Fassin, D. (2013 [2011]) Enforcing Order: An Ethnography of Urban Policing. Cambrigde: Polity Press.

Grahame, P. (1998). Ethnography, Institutions, and the Problematic of the Everyday World. Human Studies, 21, 347-360.

Griffith, A. y Smith, D. (2004). Mothering for schooling (Critical social thought). London: Routledge.

Griffith, A. (1995). Coordinating family and school: mothering for schooling. Education Policy Analysis Archives, 3(1), 1-13.

Haraway, D. (1988). Situated knowledges. The science question in fe- 
minism and the privilege of partial perspective. Feminist Studies, 14(3), 575-599.

Harding, S. (1986). The science question in feminism. Ithaca: Cornell University Press.

Hevia, F. (2011). La difícil articulación entre políticas universales y programas focalizados. Etnografía institucional del programa Bolsa Familia de Brasil. Gestión y Política Pública, XX(2), 331379 .

Hurtado, E. (2005). El oficio de la etnografía política. Diálogo con Javier Auyero. Íconos. Revista de ciencias sociales, 22, 109-126.

Imilán, W., Jirón, P. e Iturra, L. (2015). Más allá del barrio: habitar Santiago en la movilidad cotidiana. Antropologías del Sur, 3, 87103.

Iturra, L. (2014). ¿Dónde termina mi casa? Mirando el hábitat residencial desde la noción de experiencia. Revista INVI, 29(81), 221-248.

Jirón, P. (2011), On becoming "la sombra/the shadow", en M. Buscher, J. Urry y K. Witchger (Eds). Mobile Methods. New York: Routledge.

Jirón, P. e Imilan, W. (2016). Observando juntos en movimiento: posibilidades, desafíos o encrucijadas de una etnografía colectiva. Alteridades, 26(2), 51-64.

Jirón, P. y Mansilla, P. (2014). Las consecuencias del urbanismo fragmentador en la vida cotidiana de habitantes de la ciudad de Santiago de Chile. Revista EURE, 4(121), 5-28

Jirón, P., Imilán, W. e Iturra, L. (2016). Relearning to travel in Santiago: the importance of mobile place-making and travelling know-how. Cultural Geographies, 23(4), 599 - 614.

Jirón, P. e Imilán, W. (2015) Embodying flexibility: experiencing labour flexibility through urban daily mobility in Santiago de Chile. Mobilities, 10(1), 119-135.

Luken, P. \& Vaughan, S. (2015). Introduction to the special issue: new scholarship in Institutional Ethnography. The Journal of Sociology \& Social Welfare, 42(2), 3-11.

Medeiros, L. y Álvarez, P. (2016), Los trastornos mentales comunes y la medicalización: una perspectiva a partir de la etnografía institucional. Revista San Gregorio, 12, 7-15. 
Ministerio de Vivienda y Urbanismo (2011). Manual de Procedimiento del Programa de Recuperación de Barrios. Recuperado de http:/ / www.minvu.cl.

Montero, S. (2016). Worlding Ciclovia. From Urban Experiment to International "Best Practice". Latin American Perspectives, 44(2), 111-131.

Montero, S. (2017). Study tours and inter-city policy learning: Mobilizing Bogotá's transportation policies in Guadalajara. Environment and Planning A: Economy and Space, 49(2), 332-350.

Ng, S., Stooke, R., Regan, S., Hibbert, K., Schryer, C., Phelan, S. \& Lingard, L. (2013). An institutional ethnography inquiry of health care work in special education: a research protocol. International Journal of Integrated Care, 13, 1-11.

Nivar, C. e Hiciano, J. (2017). Prácticas institucionales de las Juntas Locales de Protección y Restitución de los Derechos de los niños, niñas $y$ adolescentes (NNA): Una etnografía institucional. (Tesis de Magíster, Pontificia Universidad Católica Madre y Maestra, Santo Domingo, República Dominicana). Recuperado de http:/ / hdl.handle.net/20.500.12060/1838

O'Neill, B. (1998). Institutional Ethnography: Studying Institutions from the Margins. The Journal of sociology $\mathcal{E}$ Social Welfare, 25(4), 127-144.

Paulsen, J. (2015). Captured by care: An institutional ethnography on the work of being in a rehabilitation process in Norway. The Journal of Sociology \& Social Care, 42(2), 13-29.

Rankin, J. \& Campbell, M. (2009). Institutional Ethnography (IE), Nursing Work and Hospital Reform: IE's Cautionary Analysis. Forum Qualitative Sozialforschung / Forum: Qualitative Social Research, 10(2).

Rankin, J. \& Campbell, M. (2006). Managing to Nurse: Inside Canada's Health Care Reform. Toronto: University of Toronto Press.

Rankin, J. (2017a). Conducting analysis in Institutional Ethnography: analytical work prior to commencing data collection. International Journal of Qualitative Method, 16, 1-9.

Rankin, J. (2017b). Conducting Analysis in Institutional Ethnography: Guidance and cautions. International Journal of Qualitative Method, 16, 1-11.

Restrepo, O. (2014). Análisis sociopolítico de los derechos sexuales y repro- 
ductivos (DSR) de adolescentes en la formación médica. Una etnografía institucional (EI) del proceso de renovación curricular del programa del pregrado médico en la Universidad de Antioquía (1997-2013). Tesis de doctorado en ciencias sociales, Universidad de Antioquía, Colombia. Recuperado de http:/ / hdl.handle.net/10495/3816

Rosabal-Coto, G. (2016). La versión de "El Sistema" en Costa Rica: una perspectiva desde la etnografía postcolonial institucional. Epistemus, 4(2), 84-111.

Shore, C. (2000). Building Europe: The cultural politics of European integration. London: Routledge.

Smith, D. (1974). Women's Perspective as a Radical Critique of Sociology. Sociological Inquiry, 44(1), 7-13.

Smith, D. (1987). The everyday world as problematic. A Feminist sociology. Boston: Northeastern University Press.

Smith, D. (1990). The Conceptual Practices of Power: A Feminist Sociology of Knowledge. Toronto: University of Toronto Press.

Smith, D. (1992). Sociology from women's experience: A reaffirmation. Sociological Theory, 10(1), 88-98.

Smith, D. (1997). Comment on Hekman's “Truth and Method: Feminist Standpoint Theory Revisited". Signs, 22(2), 392-398.

Smith, D. (2005). Institutional Ethnography. A sociology for people. Oxford: Altamira Press.

Smith, D. (Ed.). (2006). Institutional Ethnography as practice. Oxford: Rowman \& Littlefield Publishers.

Spinney, J. (2011). A chance to catch a breath: Using mobile video ethnography in cycling research. Mobilities, 6(2), 161-182.

Torres, F., Herrera, F., Vizcarra, I. y Lutz B. (2015). Etnografía institucional del proyecto estratégico de seguridad alimentaria (PESA) en una comunidad Mazahua. Nueva Antropología, XXVIII(82), 51-81.

Vázquez, G. (2017). La etnografía institucional: una alternativa metodológica en la investigación educativa. Revista Latinoamericana de Estudios Educativos, XLVII(2), 115-136.

Vizcarra-Bordi, I. y Farfán-González, F. (2012). El programa “Verte sano y regresa sano": lecciones de una etnografía institucional en el Estado de México. Ruris, 6(2), 123-154.

Wright, U. (2003). Institutional Ethnography: a tool for merging research and 
practice. Midwest Research to Practice Conference in Adult, Continuing, and Community Education. The Ohio State University, Columbus.

Wright, U. y Rocco, T. (2007). Institutional Ethnography: a tool for interrogating the institutional and political conditions of individual experience. Adult Education Research Conference, Halifax, Canada. Recuperado de: http://newprairiepress. org/aerc/2007/papers/108.

Yáñez, S. (2010). El seno de la paradoja: un recorrido por los inconstantes discursos de las políticas del amamantamiento. Temas de mujeres, 6(6), 116-127. 